Canadian

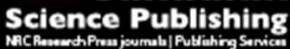

Applied Physiology, Nutrition, and Metabolism Physiologie appliquée, nutrition et métabolisme

\title{
Effects of phosphatidic acid supplementation on muscle thickness and strength in resistance-trained men
}

\begin{tabular}{|r|l|}
\hline Journal: & Applied Physiology, Nutrition, and Metabolism \\
\hline Manuscript ID & apnm-2016-0564.R1 \\
\hline Manuscript Type: & Article \\
\hline Date Submitted by the Author: & 21-Nov-2016 \\
\hline Complete List of Authors: & $\begin{array}{l}\text { Gonzalez, Adam; Hofstra University, Health Professions } \\
\text { Sell, Katie; Hofstra University } \\
\text { Ghigiarelli, Jamie; Hofstra University } \\
\text { Kelly, Christopher; Hofstra University, Health Professions } \\
\text { Shone, Edward; Hofstra University, Health Professions } \\
\text { Accetta, Matthew; Hofstra University, Health Professions } \\
\text { Baum, Jamie; Hofstra University, Health Professions } \\
\text { Mangine, Gerald; Kennesaw State University }\end{array}$ \\
\hline Keyword: & $\begin{array}{l}\text { muscle growth, resistance training < exercise, nutritional supplement, } \\
\text { ergogenic aid, phospholipid }\end{array}$ \\
\hline \multicolumn{2}{|l}{} \\
\hline
\end{tabular}


Effects of phosphatidic acid supplementation on muscle thickness and strength in resistance-trained men

\author{
Adam M. Gonzalez ${ }^{1}$, Katie M. Sell ${ }^{1}$, Jamie J. Ghigiarelli ${ }^{1}$, Christopher F. Kelly ${ }^{1}$, \\ Edward W. Shone ${ }^{1}$, Matthew R. Accetta ${ }^{1}$, Jamie B. Baum ${ }^{1}$, and Gerald T. Mangine ${ }^{2}$ \\ ${ }^{1}$ Department of Health Professions, Hofstra University, Hempstead, NY, USA \\ ${ }^{2}$ Department of Exercise Science and Sport Management, Kennesaw State University, \\ Kennesaw, GA, USA
}

Running head: Phosphatidic acid supplementation

\title{
Corresponding Author:
}

Adam M. Gonzalez, Ph.D.

Department of Health Professions

Hofstra University

Hempstead, NY 11549

516-463-5224

Adam.Gonzalez@hofstra.edu 


\begin{abstract}
The purpose of this study was to investigate the effects of phosphatidic acid (PA) supplementation on muscle thickness and strength following an 8-week supervised resistance-training program. Fifteen resistance trained men $(22.8 \pm 3.5 \mathrm{y} ; 80.6 \pm 8.7 \mathrm{~kg}$; $178.1 \pm 5.6 \mathrm{~cm} ; 14.6 \pm 8.8 \%$ body fat) were randomly assigned to a group that either consumed $750 \mathrm{mg}$ of PA or a placebo (PL). Testing was carried out before (PRE) and after (POST) training/supplementation for muscle thickness and strength. Muscle thickness of the rectus femoris (RF), vastus lateralis (VL), biceps brachii (BB), and triceps brachii (TB) muscles were measured via ultrasonography along with one repetition maximum (1RM) of squat, deadlift, and bench press. Analysis of covariance, using PRE values as the covariate, did not reveal any group differences for measures of muscle thickness in the RF (PA: $3.6 \pm 5.2 \%$; PL: $3.2 \pm 4.2 \%, \mathrm{p}=0.97)$, VL (PA:

$23.4 \pm 18.1 \%$, PL: $12.5 \pm 15.4 \%, p=0.37), B B(P A: 3.7 \pm 6.4 \%$, PL: $9.6 \pm 12.4 \%, p=0.86)$, or TB (PA: $15.1 \pm 17.9 \%$, PL: $10.7 \pm 19.3 \%, \mathrm{p}=0.79$ ). Likewise, no group differences were observed in changes in squat (PA: $8.4 \pm 4.1 \%$, PL: $8.1 \pm 4.2 \%, \mathrm{p}=0.79$ ), deadlift (PA: $10.1 \pm 10.1 \%$, PL: $8.9 \pm 9.5 \%, \mathrm{p}=0.66$ ), or bench press (PA: $5.7 \pm 5.5 \%$, PL: $5.1 \pm 3.0 \%$, $\mathrm{p}=0.76$ ) exercises. Collectively, however, all participants experienced significant $(p<0.05)$ improvements in each measure of muscle thickness and strength. Results of this study suggest that PA supplementation, in combination with a 3 days $\cdot \mathrm{week}^{-1}$ resistance- $^{-}$ training program for 8-weeks, did not have a differential effect compared to PL on changes in muscle thickness or 1RM strength.
\end{abstract}

Keywords: muscle growth; resistance training; nutritional supplement; ergogenic aid; phospholipid 


\section{INTRODUCTION}

Increasing skeletal muscle size and strength has several important implications ranging from disease prevention to increasing sports performance (Egan and Zierath 2013). Muscle mass accretion is largely governed by the relationship between muscle protein synthesis (MPS) and breakdown, whereby muscle hypertrophy occurs if protein synthesis exceeds breakdown. Moreover, MPS appears to be regulated by the multiprotein phosphorylation cascade known as the mammalian/mechanistic target of rapamycin complex 1 (mTORC1) (Goodman 2014). Activation of mTORC1 signaling has been shown to be stimulated by several inputs, including growth factors, energy status, amino acids, and mechanical stimuli (Bond 2016). Several studies have also indicated that mTORC1 signaling is required for mechanically-induced increases in MPS and subsequent hypertrophy (Bodine et al. 2001; Drummond et al. 2009; Gundermann et al. 2014).

Resistance exercise is known to be a major regulator for promoting MPS and muscle mass accretion (Gonzalez et al. 2016). Although the exact mechanism underlying increased mTORC1 activation has not been fully elucidated, resistance exercise has been suggested to promote mTORC1 activation by increasing the abundance of the diacylglycerophospholipid, phosphatidic acid (PA) (Hornberger et al. 2006; Willoughby 2015). Along with serving as a major constituent of cell membranes and a precursor for the biosynthesis of other lipids, PA is also suggested to act as an intracellular lipid second messenger, which mediates protein signaling activity (Shad et al. 2015). PA appears to be a direct regulator of resistance exercise-induced mTORC1 signaling, acting through distinct mechanisms that converge on the protein kinase, mammalian/mechanistic target 
of rapamycin (mTOR) (Shad et al. 2015). It has been suggested that PA can bind to the FKBP12-rapamycin binding (FRB) domain of mTOR, and directly activate mTOR kinase activity (Yoon et al. 2011; You et al. 2012). Therefore, the combination of resistance training and exogenous PA supplementation may up-regulate parallel pathways that converge on mTOR, thereby augmenting MPS and, thus, promote muscle hypertrophy over time (Shad et al. 2015).

Several studies have shown that administration of PA promotes an increase in mTORC1 activation in vitro and in vivo (Ávila-Flores et al. 2005; Joy et al. 2014; Mobley et al. 2015; Tang et al. 2006; You et al. 2014). However, few studies have investigated the effects of PA supplementation in humans (Andre et al. 2016; Escalante et al. 2016; Joy et al. 2014). Joy et al. (2014) observed greater hypertrophy and strength gains following PA supplementation $\left(750 \mathrm{mg} \cdot \mathrm{day}^{-1}\right)$ and 8 weeks of resistance training compared to training alone. Utilizing a similar design, Escalante and colleagues (Escalante et al. 2016) also demonstrated greater hypertrophy and strength gains following PA supplementation. However, the $750 \mathrm{mg}$ dose of PA was administered as part of a multi-ingredient supplement also containing leucine, $\beta$-hydroxy- $\beta$ methylbutyrate (HMB), and Vitamin D3. In contrast, Hoffman et al. (2012) found PA supplementation $\left(750 \mathrm{mg} \cdot \mathrm{day}^{-1}\right)$ to have a likely, but not statistically significant, benefit on hypertrophy and strength outcomes following 8 weeks of unsupervised resistance training. Likewise, Andre et al. (2016) found lower dosages of PA (375 and $250 \mathrm{mg} \cdot$ day $^{-}$ $\left.{ }^{1}\right)$ to have a likely, but not statistically significant, benefit on hypertrophy and strength outcomes when combined with an 8-week resistance-training program. Given the equivocal findings of these studies, additional research is needed to examine the 
effectiveness of PA supplementation. Thus, the purpose of this study was to examine the efficacy of $750 \mathrm{mg}$ of PA on muscle thickness and strength gains in resistance-trained men.

\section{METHODS AND MATERIALS}

\section{Participants}

Initially, sixteen resistance-trained men were recruited to participate in this randomized, double-blind, placebo-controlled study. One subject voluntarily withdrew from the study due to time constraints. Fifteen participants $(22.8 \pm 3.5 \mathrm{y} ; 80.6 \pm 8.7 \mathrm{~kg}$; $178.1 \pm 5.6 \mathrm{~cm} ; 14.6 \pm 8.8 \%$ body fat) completed the study protocol. All participants had a minimum of 1 year of resistance training experience and were currently carrying out strength training sessions at least 2 days per week. All participants were free of any physical limitations that may affect performance, and all participants were free of medications, dietary supplements, and performance enhancing drugs known to increase muscle mass and/or performance, as determined by a health and activity questionnaire. While enrolled in the study, participants were required to abstain from consuming any other dietary supplements and abstain from training outside of the prescribed protocol (other than activities of daily living). Following an explanation of all procedures, risks, and benefits, each participant provided his informed consent prior to participation in this study. The research protocol was approved by the Hofstra University Institutional Review Board prior to participant enrollment.

\section{Study protocol}

Participants were matched based on baseline measures of body mass and randomly assigned to one of two treatment groups: PA or placebo (PL). Participants 
reported to the Human Performance Laboratory for ultrasonography and strength assessments within 7 days prior to the first dose of supplement and beginning of the resistance-training program (PRE), and within 7 days following the 8-week intervention (POST). Ultrasonography assessments were performed approximately $24 \mathrm{~h}$ prior to all strength measures, and each testing session occurred following 72 hours of rest from exercise (other than activities of daily living) to allow proper recovery and ensure that training-induced muscle swelling did not obscure the results (Kristiansen et al. 2014).

\section{Supplement protocol}

The PA group received 6 capsules per day containing $750 \mathrm{mg}$ of PA. The PL group received 6 capsules per day containing 2.4 grams of psyllium husk fiber. The capsules were similar in appearance (size and color). The dosing strategy was similar to a previous study investigating the effects of PA supplementation (Joy et al. 2014). On resistance-training days, participants consumed 3 capsules of their respective supplement 30 minutes prior to resistance training and 3 capsules immediately following resistance training along with $20 \mathrm{~g}$ of hydrolyzed collagen protein (Table 1; Perfect Supplements, Coventry, RI, USA) mixed with $350 \mathrm{ml}$ water. The protein supplement was provided by the research team to standardize post-exercise meals between groups, and hydrolyzed collagen was chosen as an incomplete protein source low in leucine in order to potentially minimize the impact on the PA supplement. On non-resistance-training days, participants consumed 3 capsules of their respective supplement with breakfast and 3 capsules with dinner. Participants were provided with an allotted amount of capsules at the start of each week. To ensure compliance, participants were required to return their empty bag at the end of each week. Thus, any remaining capsules were deemed as non- 
compliance.

\section{***INSERT TABLE 1 HERE***}

\section{Resistance-training program}

All participants completed an 8-week resistance-training program under the direct supervision of certified strength and conditioning specialists (CSCS). Resistance training occurred three days per week with 48-72 hours between sessions (Table 2). Four sets of each exercise were performed using a load that allowed the participant to complete a 10RM. A 2-min rest interval was allowed between sets and exercises. Exercises and repetition schemes remained the same for all 8 weeks. All participants were required to perform 10 repetitions with their prescribed weight. In the event that a participant reached muscular failure, a researcher assisted with the completion of the exercise. Training loads for a give exercise were increased if the participant could complete all 4 sets of 10 repetitions with proper technique prior to muscular failure.

***INSERT TABLE 2 HERE***

\section{Muscle thickness assessment}

Noninvasive skeletal muscle ultrasound images were collected from the dominant thigh and arm locations of all participants using B-mode ultrasound imaging with a 12MHz linear probe (VSCAN, General Electric Medical Systems, Milwaukee, WI, USA). Each participant laid supine on the examination table for a minimum of 15 min to allow fluid shifts to occur before images were collected (Berg et al. 1993). Prior to image collection, all anatomical locations of interest were identified using standardized landmarks for the rectus femoris (RF), vastus lateralis (VL), triceps brachii (TB), and biceps brachii (BB) muscles (Mangine et al. 2015). A single technician performed 
landmark measurements and obtained images in duplicate for all participants. The landmark for the RF was $50 \%$ of the distance from the anterior, inferior suprailiac crest and the proximal border of the patella. The landmark for the VL was $50 \%$ of the distance from the most prominent point of the greater trochanter of the femur to the inferior border of the patella. Landmark identification of the TB required the participant to straddle the examination table and internally rotate their dominant shoulder, flex the elbow, and rest their dominant hand upon their thigh. The specific landmark for the TB was identified at $60 \%$ of the distance from the acromion process of the scapula to the lateral epicondyle of elbow. Lastly, the landmark for the BB required the participant to lay supine with hands supinated. The specific landmark for the BB was identified at $60 \%$ of the distance from the acromion process of the scapula to the lateral epicondyle of the humerus.

Following application of a water-soluble gel, the ultrasound probe was oriented longitudinally in the sagittal plane parallel to the muscle with the center of the probe positioned on the established landmark. The probe was placed perpendicular to the tissue interface without depressing the skin. Muscle thickness was determined as the distance between the subcutaneous adipose tissue and intermuscular interface. Prior to the investigation, $\mathrm{ICC}_{3, \mathrm{~K}}, \mathrm{SEM}$, and MD values for muscle thickness of the $\mathrm{RF}\left(\mathrm{ICC}_{3, \mathrm{~K}}=0.99\right.$, $\left.\mathrm{SEM}_{3, \mathrm{~K}}=0.05, \mathrm{MD}=0.13 \mathrm{~cm}\right), \mathrm{VL}\left(\mathrm{ICC}_{3, \mathrm{~K}}=0.95, \mathrm{SEM}_{3, \mathrm{~K}}=0.07, \mathrm{MD}=0.20 \mathrm{~cm}\right), \mathrm{TB}$ $\left(\mathrm{ICC}_{3, \mathrm{~K}}=0.93, \mathrm{SEM}_{3, \mathrm{~K}}=0.13, \mathrm{MD}=0.34 \mathrm{~cm}\right)$, and $\mathrm{BB}\left(\mathrm{ICC}_{3, \mathrm{~K}}=0.97, \mathrm{SEM}_{3, \mathrm{~K}}=0.18\right.$, $\mathrm{MD}=0.50 \mathrm{~cm}$ ) were determined in 10 healthy college-aged males using methodology described above. 


\section{Strength assessment}

During each testing session, participants performed a one-repetition maximum (1RM) strength test for the barbell back squat, deadlift, and bench press exercises using a standard protocol (Hoffman 2006). Prior to maximal strength testing, participants performed a standardized warm-up consisting of 10 body weight squats, 10 body weight walking lunges, 10 dynamic walking hamstring stretches, 10 dynamic walking quadriceps stretches, and 10 body weight pushups. For each exercise, participants performed two warm-up sets using a resistance of approximately $40-60 \%$ of estimated $1 \mathrm{RM}$ for $6-10$ repetitions and $60-80 \%$ of estimated $1 \mathrm{RM}$ for $3-5$ repetitions, respectively. The weight was then increased conservatively, and the participant attempted to lift the weight for one repetition. A 3-5 minute rest period was provided between each attempt. This procedure continued until the participant failed to complete the lift. The 1RM was recorded as the maximum weight that the participant was able to lift for one repetition. All testing was completed under the supervision of a CSCS.

A successful lift in the squat required the participant to descend to a thigh parallel position defined by the trochanter head of the femur reaching the same horizontal plane as the superior border of the patella. An investigator located lateral to the participant provided an "Up!" signal indicating when proper range of motion had been achieved.

Deadlifts were performed with a conventional shoulder width stance, and a successful lift required the participant to achieve an erect body posture with the knees straightened and shoulders retracted upon completion of the movement. Bench press was performed with a conventional shoulder width grip, and a successful lift required the participant to lower the bar until lightly touching the chest (i.e., subjects were not permitted to bounce the bar 
off of the chest) prior to lifting the bar back to a straight-arm position with the hips and feet remaining in contact with the bench and floor, respectively, throughout the lift (Hoffman 2006).

\section{Dietary analysis}

Participants were advised to maintain their normal diet and to avoid taking any supplements other than those provided in the study. To assess the potential confounding impact of dietary intake, self-reported food records were collected during the first (week 1) and final week (week 8) of the study. Participants were instructed on how to properly complete a three-day dietary recall log to include all food items and their respective portion sizes that were consumed. Dietary analysis software $\left(\right.$ MyFitnessPal $\left.^{\circledR}\right)$ was used to analyze dietary recalls to assess potential differences in total energy and macronutrient intakes between groups.

\section{Statistical analysis}

To identify differences between the experimental conditions on changes in muscle size and strength, an analysis of covariance (ANCOVA) was performed on all measures collected at POST. Associated values collected at PRE were used as the covariate to eliminate the possible influence of initial score variances on the outcomes. Following any significant F-ratio, a paired-samples t-test was used to determine if significant difference existed between measures collected prior to and immediately following 8 weeks of training. All between group differences were further analyzed using effect sizes

$\left(\eta^{2}:\right.$ partial eta squared). Interpretations of effect size were evaluated (Green et al. 2000) at the following levels: small effect (0.01-0.059), medium effect (0.06-0.139) and large effect $(>0.14)$. A criterion alpha level of $p<0.05$ was used to determine statistical 
significance. All data are reported as mean \pm standard deviation. Statistical Software (V. 21.0, SPSS Inc., Chicago, IL) was used for all analyses.

\section{RESULTS}

\section{Participant compliance, reported side effects \& dietary analysis}

Resistance training and supplement compliance was high (both $>98 \%$ ) with no differences between groups. PA and PL supplements were well tolerated and no adverse side effects were reported. No significant differences $(\mathrm{p}>0.05)$ were noted for caloric intake (2260.1 $\pm 736.3 \mathrm{kcal}$ vs. $2111.1 \pm 740.8 \mathrm{kcal})$, carbohydrate intake $(234.3 \pm 69.1 \mathrm{~g}$ vs. $230.4 \pm 112.2 \mathrm{~g})$, fat intake $(90.3 \pm 42.1 \mathrm{~g}$ vs. $82.5 \pm 33.0 \mathrm{~g})$, protein intake $(121.7 \pm 40.0 \mathrm{~g}$ vs. $121.2 \pm 31.4 \mathrm{~g})$, or relative protein intake $(1.5 \pm 0.5 \mathrm{~g}$ vs. $1.6 \pm 0.6 \mathrm{~g})$ during week 1 between PA and PL, respectively. Additionally, no significant differences $(\mathrm{p}>0.05)$ were noted when comparing the participants' nutritional intake during week 1 and week 8 of the study.

\section{Muscle thickness and strength}

When using PRE values as the covariate, no group differences were observed for any measure of muscle thickness or strength following 8-weeks of resistance training. Collectively, significant improvements occurred in both groups for all measures of muscle thickness $(p<0.05)$ and strength $(p<0.001)$. The changes in muscle thickness and strength following 8 weeks of resistance training are presented in Table 3.

$$
\text { ***INSERT TABLE } 3 \text { HERE*** }
$$

\section{DISCUSSION}

In the present study, we investigated the effects of PA supplementation on muscle thickness and strength following an 8-week supervised resistance-training program in 
resistance-trained men. The results of this study indicate that a daily dose of $750 \mathrm{mg}$ of PA did not have a differential effect compared to PL on increasing muscle thickness of RF, VL, BB, and TB or maximal strength in the barbell back squat, deadlift, and bench press exercises.

The literature to date indicates that direct binding of PA to mTOR activates downstream mTORC1 signaling (Hornberger et al. 2006; Willoughby 2015). Thus, it is tenable that increasing the presence of PA within skeletal muscle through oral supplementation, in conjunction with a mechanical stimulus, would augment mTORC1 signaling and promote greater increases in muscle hypertrophy. However, to date, no study has examined the effectiveness of PA supplementation in combination with resistance exercise on increasing intramuscular concentrations of PA or augmenting mTORC1 activity in human skeletal muscle. Nevertheless, previous studies have investigated the efficacy of PA supplementation in combination with resistance training on changes in body composition, muscle mass, and strength in trained men (Andre et al. 2016; Escalante et al. 2016; Hoffman et al. 2012; Joy et al. 2014). Hoffman et al. (2012) and Andre et al. (2016) implemented a similar periodized 4 days $\cdot \mathrm{week}^{-1} \mathrm{resistance}^{-}$ training program split into two upper- and two lower-extremity sessions per week. In both Hoffman et al. (2012) and Andre et al. (2016), magnitude-based inferences demonstrated likely beneficial effects of PA on muscle growth and strength, while modern statistics found no statistical interactions between groups. However, the participants in Hoffman et al. (2012) were not supervised during the resistance-training program, which could potentially confound the findings, and a lower dose which may not have been sufficient to elicit significant results was utilized by Andre et al. (2016). 
Alternatively, Joy et al. (2014) and Escalante et al. (2016) implemented 8 weeks of supervised resistance training utilizing a 3 day ${ }^{\cdot} \mathrm{week}^{-1}$ undulating single-set resistancetraining periodization program and found PA supplementation $\left(750 \mathrm{mg} \cdot \mathrm{day}^{-1}\right)$ to promote significantly greater hypertrophy and strength gains. Joy et al. (2014) demonstrated greater increases in lean body mass, RF cross sectional area, and 1RM leg press strength as a result of PA supplementation, while no differences were noted for bench press strength. Escalante et al. (2016) demonstrated greater increases in lean body mass, 1RM leg press strength, and 1RM bench press strength, while no differences were noted for thigh muscle mass, vertical jump, agility, or peak power output. However, the supplement administered in Escalante et al. (2016) included a proprietary blend of Lleucine, HMB, and vitamin D3 which makes it difficult to discern if the results were attributable to PA.

In the current investigation, no significant differences in muscle architecture or strength measures were found following PA supplementation compared to PL. Similar to Hoffman et al. (2012), we found no statistical interactions between groups for VL thickness, squat strength, and bench press strength. It is unclear why discrepancies exist in study outcomes following PA supplementation in conjunction with an 8-week resistance-training program. With the exception of Andre et al. (2016), previous investigations provided resistance-trained men with $750 \mathrm{mg}^{-\mathrm{day}^{-1}}$ prior to and immediately following resistance training along with a collagen protein source in resistance-trained men. Andre et al. (2016) had resistance-trained men ingest lower doses of PA (375 and $250 \mathrm{mg} \cdot \mathrm{day}^{-1}$ ) 60 minutes prior to resistance exercise and did not provide a post-workout collagen protein supplement. Other potential discrepancies include 
exercise supervision, resistance-training program design, dietary adherence, exercise selection for assessing maximal strength (e.g., leg press vs. back squat), timing of supplement ingestion, methods of assessing changes in muscle architecture and body composition (e.g., dual-energy X-ray absorptiometry vs. ultrasound), and training status of study participants.

Considering that the mTOR signaling pathway was not examined in this study or any of the previous human studies involving resistance training and PA supplementation, only speculation can be made on the mechanisms that may contribute to an overall effect on muscular adaptation. While in vitro studies demonstrate an upregulation of mTOR signaling following PA administration (Ávila-Flores et al. 2005; Joy et al. 2014; Tang et al. 2006; You et al. 2014), it is difficult to draw direct implications to humans or substantiate the role that PA supplementation may have in conjunction with resistance exercise. Furthermore, although the stimulation of MPS appears to require mTORC1 activation, dissociation between anabolic signaling and MPS may exist, whereby mTORC1 activation is not consistently associated with MPS rates (Greenhaff et al. 2008; Mitchell et al. 2015). Therefore, upregulation of anabolic signaling in vitro may provide little, if any, explanation towards the dynamic process of muscle hypertrophy in humans (Damas et al. 2015). Additional research is warranted to investigate the pharmacokinetics of different orally administered PA dosages and the subsequent impact on intramuscular mTORC1 signaling and MPS when ingested in combination with resistance exercise in humans. Likewise, additional long-term randomized controlled trials in humans are necessary to determine the efficacy of PA as a solution to promote skeletal muscle anabolism in a variety of populations. 
In conclusion, in the current investigation, PA supplementation did not appear to further augment muscle thickness or strength following 8 weeks of resistance training in resistance-trained men. Supplementation of $750 \mathrm{mg}$ PA for 8 weeks in conjunction with a supervised resistance-training program did not have a differential effect compared to PL on changes in muscle thickness of RF, VL, BB, and TB. Additionally, no additive effect of PA supplementation was observed for 1RM strength in the barbell back squat, deadlift, and bench press exercises.

\section{ACKNOWLEDGMENTS}

The authors wish to thank all participants who gave of their time and effort to participate in this study. We are also grateful to Noah Siegel at Siege Athletics LLC and Mike Nunziato at Total Nunziato Training LLC for providing the resources and equipment necessary to make this study possible. This research was supported by the Hofstra University School of Education Faculty Research Grant.

\section{CONFLICT OF INTEREST}

The authors declare no conflict of interest. 


\section{REFERENCES}

Andre, T.L., Gann, J.J., McKinley-Barnard, S.K., Song, J.J., and Willoughby, D.S. 2016. Eight weeks of phosphatidic acid supplementation in conjunction with resistance training does not differentially affect body composition and muscle strength in resistance-trained men. J. Sports Sci. Med. 15(3): 532-539.

Ávila-Flores, A., Santos, T., Rincón, E., and Mérida, I. 2005. Modulation of the mammalian target of rapamycin pathway by diacylglycerol kinase-produced phosphatidic acid. J. Biol. Chem. 280(11): 10091-10099.

Berg, H., Tedner, B., and Tesch, P. 1993. Changes in lower limb muscle cross - sectional area and tissue fluid volume after transition from standing to supine. Acta Physiol. Scand. 148(4): 379-385.

Bodine, S.C., Stitt, T.N., Gonzalez, M., Kline, W.O., Stover, G.L., Bauerlein, R., et al. 2001. Akt/mTOR pathway is a crucial regulator of skeletal muscle hypertrophy and can prevent muscle atrophy in vivo. Nature Cell Biol. 3(11): 1014-1019.

Bond, P. 2016. Regulation of mTORC1 by growth factors, energy status, amino acids and mechanical stimuli at a glance. J. Int. Soc. Sports Nutr. 13(8): 1-11.

Damas, F., Phillips, S., Vechin, F.C., and Ugrinowitsch, C. 2015. A review of resistance training-induced changes in skeletal muscle protein synthesis and their contribution to hypertrophy. Sports Med. 45(6): 801-807.

Drummond, M.J., Fry, C.S., Glynn, E.L., Dreyer, H.C., Dhanani, S., Timmerman, K.L., et al. 2009. Rapamycin administration in humans blocks the contraction-induced increase in skeletal muscle protein synthesis. J. Physiol. 587(7): 1535-1546.

Egan, B. and Zierath, J.R. 2013. Exercise metabolism and the molecular regulation of skeletal muscle adaptation. Cell Metab. 17(2): 162-184.

Escalante, G., Alencar, M., Haddock, B., and Harvey, P. 2016. The effects of phosphatidic acid supplementation on strength, body composition, muscular endurance, power, agility, and vertical jump in resistance trained men. J. Int. Soc. Sports Nutr. 13(24): 1-13.

Gonzalez, A.M., Hoffman, J.R., Stout, J.R., Fukuda, D.H., and Willoughby, D.S. 2016. Intramuscular anabolic signaling and endocrine response following resistance exercise: Implications for muscle hypertrophy. Sports Med. 46(5): 671-685.

Goodman, C.A. 2014. The role of $\mathrm{mTORC} 1$ in regulating protein synthesis and skeletal muscle mass in response to various mechanical stimuli. Rev. Physiol Bioch. Pharm. 166: 43-95.

Green, S., Salkind, N., and Akey, T. 2000. Methods for controlling type I error across multiple hypothesis tests. Using SPSS for Windows: Analysing and Understanding Data. 395-396.

Greenhaff, P.L., Karagounis, L., Peirce, N., Simpson, E.J., Hazell, M., Layfield, R., et al. 2008. Disassociation between the effects of amino acids and insulin on signaling, ubiquitin ligases, and protein turnover in human muscle. Am. J. Physiol. Endocrinol. Metab. 295(3): E595-E604.

Gundermann, D.M., Walker, D.K., Reidy, P.T., Borack, M.S., Dickinson, J.M., Volpi, E., et al. 2014. Activation of mTORC1 signaling and protein synthesis in human muscle following blood flow restriction exercise is inhibited by rapamycin. Am. J. Physiol. Endocrinol. Metab. 306(10): E1198-E1204. 
Hoffman, J. 2006. Norms for fitness, performance, and health. Human Kinetics. Champaign, IL.

Hoffman, J.R., Stout, J.R., Williams, D.R., Wells, A.J., Fragala, M.S., Mangine, G.T., et al. 2012. Efficacy of phosphatidic acid ingestion on lean body mass, muscle thickness and strength gains in resistance-trained men. J. Int. Soc. Sports Nutr. 9(47): 1-7.

Hornberger, T., Chu, W., Mak, Y., Hsiung, J., Huang, S., and Chien, S. 2006. The role of phospholipase D and phosphatidic acid in the mechanical activation of mTOR signaling in skeletal muscle. Proc. Natl. Acad. Sci. U.S.A. 103(12): 4741-4746.

Joy, J.M., Gundermann, D.M., Lowery, R.P., Jäger, R., McCleary, S.A., Purpura, M., et al. 2014. Phosphatidic acid enhances mTOR signaling and resistance exercise induced hypertrophy. Nutr. Metabolism. 11(29): 1-10.

Kristiansen, M.S., Uhrbrand, A., Hansen, M., Shiguetomi - Medina, J.M., Vissing, K., et al. 2014. Concomitant changes in cross-sectional area and water content in skeletal muscle after resistance exercise. Scand. J. Med. Sci. Spor. 24(4): e260e268.

Mangine, G.T., Hoffman, J.R., Gonzalez, A.M., Townsend, J.R., Wells, A.J., Jajtner, A.R., et al. 2015. The effect of training volume and intensity on improvements in muscular strength and size in resistance-trained men. Physiol. Rep. 3(8): e12472.

Mitchell, C.J., Churchward-Venne, T.A., Cameron-Smith, D., and Phillips, S.M. 2015. What is the relationship between the acute muscle protein synthesis response and changes in muscle mass? J. Appl. Physiol. 118(4): 495-497.

Mobley, C.B., Hornberger, T.A., Fox, C.D., Healy, J.C., Ferguson, B.S., Lowery, R.P., et al. 2015. Effects of oral phosphatidic acid feeding with or without whey protein on muscle protein synthesis and anabolic signaling in rodent skeletal muscle. J. Int. Soc. Sports Nutr. 12(32): 1-11.

Shad, B.J., Smeuninx, B., Atherton, P.J., and Breen, L. 2015. The mechanistic and ergogenic effects of phosphatidic acid in skeletal muscle. Appl. Physiol. Nutr. Metab. 40(12): 1233-1241.

Tang, W., Yuan, J., Chen, X., Gu, X., Luo, K., Li, J., et al. 2006. Identification of a novel human lysophosphatidic acid acyltransferase, LPAAT-theta, which activates mTOR pathway. J. Biochem. Mol. Biol. 39(5): 626-635.

Willoughby, D.S. 2015. mTORC1 Signaling-Activated Increases in Muscle Protein Synthesis induced by leucine and Phosphatidic Acid: A Brief Review. Int. J. Food Nutr. Sci. 2(1): 1-6.

Yoon, M.-S., Sun, Y., Arauz, E., Jiang, Y., and Chen, J. 2011. Phosphatidic acid activates mammalian target of rapamycin complex 1 (mTORC1) kinase by displacing FK506 binding protein 38 (FKBP38) and exerting an allosteric effect. J. Biol. Chem. 286(34): 29568-29574.

You, J.-S., Lincoln, H.C., Kim, C.R., Frey, J.W., Goodman, C.A., Zhong, X.P., et al. 2014. The role of diacylglycerol kinase $\zeta$ and phosphatidic acid in the mechanical activation of mammalian target of rapamycin (mTOR) signaling and skeletal muscle hypertrophy. J. Biol. Chem. 289(3): 1551-1563.

You, J.S., Frey, J.W., and Hornberger, T.A. 2012. Mechanical stimulation induces mTOR signaling via an ERK-independent mechanism: implications for a direct activation of mTOR by phosphatidic acid. PloS One. 7(10): e47258. 
Table 1. Amino acid profile of hydrolyzed collagen supplement

Amino Acid

Alanine

Arginine

Aspartic acid

Cysteine

Glutamic acid

Glycine

Histidine

Hydroxyproline

Isoleucine

Leucine

Lysine

Methionine

Phenylalanine

Proline

Serine

Threonine

Tyrosine

Valine mg/serving

1956

1710

1234

8

2160

5198

148

2182

320

594

792

190

426

2754

706

382

122

484 
Table 2. Resistance training program

\begin{tabular}{ccc}
\hline$\underline{\text { Monday }}$ & $\underline{\text { Wednesday }}$ & $\underline{\text { Friday }}$ \\
\hline Barbell deadlift & Barbell bench press & Barbell back squat \\
DB reverse lunge & DB fly & Stiff-leg deadlift \\
Barbell bent over row & Seated DB shoulder press & DB skullcrusher \\
DB pullover & Barbell upright row & Barbell bicep curl \\
DB hammer curl & DB front raise & DB overhead tricep extention \\
\hline
\end{tabular}

All participants completed an 8-week resistance-training program under the direct supervision of certified strength and conditioning specialists. Resistance training occurred three days per week with 48-72 hours between sessions. Four sets of 10RM were performed for each exercise. Exercises and repetition schemes remained the same for all 8 weeks. Training loads for a give exercise were increased if the subject could lift a given weight for 4 sets of 10 repetitions with proper technique prior to muscular failure. A 2-min rest interval was allowed between sets and exercises. $\mathrm{DB}=$ dumbbell 
Table 3. Changes in muscle thickness and strength following 8 weeks of resistance training

\begin{tabular}{|c|c|c|c|c|c|c|c|c|}
\hline & \multirow[b]{2}{*}{ PRE } & \multirow[b]{2}{*}{ Covariate } & \multirow[b]{2}{*}{ POST } & \multirow[b]{2}{*}{$\mathbf{F}$} & \multirow[b]{2}{*}{ p-value } & \multirow[b]{2}{*}{$\eta^{2}$} & \multicolumn{2}{|c|}{$\begin{array}{l}\text { 95\% Confidence } \\
\text { Interval }\end{array}$} \\
\hline & & & & & & & Lower & Upper \\
\hline \multicolumn{9}{|l|}{ Muscle Thickness (cm) } \\
\hline \multicolumn{9}{|l|}{ Rectus Femoris } \\
\hline PA & $2.63 \pm 0.29$ & \multirow{2}{*}{2.56} & $2.72 \pm 0.36$ & \multirow{2}{*}{$<0.01$} & \multirow{2}{*}{0.97} & \multirow{2}{*}{$<0.01$} & 2.55 & 2.75 \\
\hline PL & $2.48 \pm 0.33$ & & $2.57 \pm 0.32$ & & & & 2.54 & 2.76 \\
\hline \multicolumn{9}{|l|}{ Vastus Lateralis } \\
\hline PA & $2.1 \pm 0.55$ & \multirow{2}{*}{1.96} & $2.61 \pm 0.83$ & \multirow{2}{*}{0.89} & \multirow{2}{*}{0.37} & \multirow{2}{*}{0.07} & 2.16 & 2.66 \\
\hline PL & $1.8 \pm 0.17$ & & $2.02 \pm 0.35$ & & & & 1.98 & 2.51 \\
\hline \multicolumn{9}{|l|}{ Biceps Brachii } \\
\hline PA & $3.67 \pm 0.38$ & \multirow{2}{*}{3.43} & $3.80 \pm 0.36$ & \multirow{2}{*}{0.03} & \multirow{2}{*}{0.86} & \multirow{2}{*}{$<0.01$} & 3.37 & 3.88 \\
\hline PL & $3.16 \pm 0.29$ & & $3.46 \pm 0.39$ & & & & 3.38 & 3.93 \\
\hline \multicolumn{9}{|l|}{ Triceps Brachii } \\
\hline PA & $2.26 \pm 0.42$ & \multirow{2}{*}{2.36} & $2.57 \pm 0.41$ & \multirow{2}{*}{0.07} & \multirow{2}{*}{0.79} & \multirow{2}{*}{0.01} & 2.37 & 2.87 \\
\hline PL & $2.48 \pm 0.33$ & & $2.73 \pm 0.31$ & & & & 2.40 & 2.94 \\
\hline \multicolumn{9}{|l|}{ One-repetition maximum ( $\mathrm{kg}$ ) } \\
\hline \multicolumn{9}{|l|}{ Squat } \\
\hline PA & $121.8 \pm 17.2$ & \multirow{2}{*}{119.5} & $131.8 \pm 17.9$ & \multirow{2}{*}{0.07} & 070 & 001 & 125.7 & 133.4 \\
\hline PL & $117.2 \pm 20.5$ & & $126.6 \pm 20.7$ & & 0.19 & 0.01 & 125.1 & 132.7 \\
\hline Deadlift & & & & & & & & \\
\hline PA & $146.4 \pm 24.3$ & 1451 & $160.1 \pm 21.9$ & 021 & 066 & ב 00 & 149.1 & 168.7 \\
\hline PL & $143.8 \pm 21.4$ & $14 J .1$ & $154.9 \pm 24.8$ & 0.21 & 0.00 & 0.02 & 146.3 & 165.8 \\
\hline Bench Press & & & & & & & & \\
\hline PA & $93.2 \pm 8.8$ & 027 & $98.3 \pm 8.0$ & 010 & 076 & $0 \Omega 1$ & 95.0 & 100.7 \\
\hline PL & $92.2 \pm 17.9$ & 92.1 & $96.8 \pm 18.1$ & 0.10 & 0.10 & 0.01 & 94.2 & 100.3 \\
\hline
\end{tabular}


